Accelerator Division

Alternating Gradient Synchrotron Department

BROOKHAVEN NATIONAL LABORATORY

Upton, New York 11973

Accelerator Division

Technical Note

AGS/AD/Tech. Note No. 441

What If Someone Were to Saw the Two BTA

Bends into Halves?

J. Niederer

August 26, 1996 


\title{
What If Someone Were to Saw the Two BTA Bends into Halves?
}

\author{
J. Niederer \\ AGS Department \\ Brookhaven National Laboratory
}

August 23, 1996

\section{Summary}

One of many possible schemes for improving the BTA is sketched. Its primary feature is to reduce dispersion in the early bending region by replacing the present pair of dipoles with four shorter ones, separated equally by quads of alternating polarities. These cells are tuned to give a region of vanishing dispersion after the bends, where alternating quads produce the relative $\mathrm{X}$ and $\mathrm{Y}$ phase advances needed for AGS acceptance. The present lattice from the Booster through quad QH4 is retained, along with the section from DH5 into the AGS. Typical operating Booster delivery parameters and AGS acceptance parameters have been used in these model studies. No Booster perturbations are involved in this particular set of tracking games.

\section{The Trial Lattice}

The intent here is to explore a relatively simple lattice geometry that makes use of the experience gained from continuing BTA studies. [1,2]. We show one such plausible lattice and resulting optics in Figure 1, along with the corresponding listing for a tracking run. For simplicity, the existing dipoles DH2 and DH3 have been halved, and $\mathrm{V}$ quads placed midway between the halves, in the usual $\mathrm{H}-\mathrm{B}-\mathrm{V}-\mathrm{B}-\mathrm{H}-\mathrm{B}-\mathrm{V}-\mathrm{B}-\mathrm{H}$ pattern. Two additional quads are involved to clamp dispersion through the bend region. The common bend angle was adjusted by about 5\% upwards to center beam in bend DH5 near the AGS entrance. The DH5 bend angle was adjusted accordingly, increased by about $15 \%$. This line of bends reaches outwards about $.5 \mathrm{~m}$ closer to the AGS than the present arrangement, using somewhat arbitrary $.6 \mathrm{~m}$ spacings among magnets. The spacing is mainly to keep the bending profile about the same as it is now. All of this pretends that the shield wall is adequately porous. Five quads, QH4, QV4, QH5, QV5, QH6, are spaced equally among these four bends. Similarly eight quads of the present QV5 type are spaced equally in the region of Q6 through Q13, two more than at present as suggested by the previous study, for a total of 19 quads. Again for simplicity, correctors, monitors, and other stuff is omitted. None of these placements or spacings are optimized. 


\section{The Matching Technique}

Matching using the BNL MAD simplex method needed a lot of guidance to deal with the 19 quad current parameters and the given entrance and exit conditions. The incoming dispersion of $2.85 \mathrm{~m}$ is high and has to be clamped down in the bend region. The existing QV1 - QH4 quad arrangement is not matched to either the BTA or the Booster optics, but is left alone here in favor of easier regions to rebuild. Initially, rather wide AGS acceptance conditions are used to get the parameter search into a reasonable region of parameter space. Amplitudes are clamped strongly at Beta max $<25 \mathrm{~m}$. Dispersion limits are then successively reduced to Dmax $<2.5 \mathrm{~m}$, which leads to a gently sloping dispersion that crosses zero somewhat after the bends. Then the AGS acceptance windows are reduced in a series of iterations. The final alphas and betas are easily reached, but the dispersion components are not, and the apparent convergence usually has to be watched. The iterations are helped by a feature added to the FEndMatch command that optionally writes a file of the set of final fitted parameter values. These values, along with adjustments to constraints and step sizes, are then input to the next iteration.

\section{Results}

This class of fit has a proper minimum, which is rather flat and has a common center among all of the quad current parameters. The fit is generally insensitive to quad current drifts. The envelopes for the orbit functions are shown in Figure 2, where each of these currents are varied in turn between two amps on either side of the fitted value. Most of the slight width observed is due to the first three quads, QV1, QH2, and QV3. This arrangement is also insensitive to incoming momentum offsets. The envelopes for a $-2 \%$ to $+2 \%$ swing in input momentum offset are shown in Figure 3 . The eventual fit noted here is a little squeezed by the beta max constraint of $<25 \mathrm{~m}$, mostly because of the way that the existing QV1 - QH4 geometry has to deal with the incoming dispersion.

The largest dispersion in this display is at the BTA entrance, set by the unfortunate choice of extraction point in the Booster. This is the limiting constraint for momentum acceptance, and possibly a source for cooking the Booster septum region. While this kind of BTA appears to handle momentum acceptance rather well, it does not eliminate the need for reducing dispersion in the Booster extraction region. Once into the BTA our four bend design permits full control of the dispersion through the bends. It further produces a desirable region of low dispersion with gentle slope downstream, which allows quads there to deal with $\mathrm{X}$ and $\mathrm{Y}$ phases without unduly prejudicing the dispersion near the AGS. The quads here can undoubtedly be better placed for this phase matching role.

This simple example points towards a more useful set of matching criteria for the actual BTA operation. The bend region should tightly contain dispersion, and slope it to reach zero in the center of the quad group located to deal with phase adjustment. Both dispersion value and slope components should appear as a formal constraint at the given point. The rest of the quads up to the AGS mainly deal with clamping the amplitudes during passage.

\section{Comments}

This kind of experience suggests including the magnet layout geometry in the matching process. In MAD, lattice geometry is computed in the group of routines that carry out the Survey command. Survey now has to be iterated through a number of 
separate runs to adjust quantities such as bend angle to lay out the beam path along an actual floor or whatever. While there is a private survey match feature attached to Cern MAD V8 [3], unfortunately it doesn't mix very easily with our simplex, for which tracking is based on our vastly improved data base. Beam line matches with their numerous free parameters have needed the far more capable simplex variant developed here. Survey matching has thus been grafted into our versions of MAD, which is easy enough because of our structure tools and much better developed matching features, inspired far too much by this BTA mess. This kind of code maintenance is in fact so straightforward that well over a thousand lines of new code for survey matching commands and constraints have been added throughout the approximately 6700 lines of the MatchF source file within a day, without affecting the present BTA match calculations. Survey matching should make it rather simple to optimize magnet placements, bend angles, and spacing for more careful beam line designs.

J. Glenn has suggested that there may be ways to play with the potential focusing capabilities of the present two main BTA dipoles, which might lead to some of the

dispersion reducing effects discussed here. This idea might be explored further in these models.

\section{References}

1. J. Niederer, BNL MAD Program Notes: BTA Lattice Matching. AGS/AD Tech Note 431. Internal Report. March, 1996.

2. J. Niederer, More BTA Lattice Matching. AGS/AD Tech Note 440. Internal Report. August, 1996.

3. H. Grote, CERN.

\section{Documents}

Unix Typesetter Format - troff / psroff Files.

\section{Host}

This Report

Reference 2

Reference 1

FMatch

Runtwiss rapt.ags.bnl.gov /usr/disc2/jn/Docum+/BTA.notes2 /usr/disc2/jn/Docum+/BTA.notes1 /usr/disc2/jn/Docum+/Match.notes /usr/disc2/jn/Docum+/Fmatch.man /usr/disc2/jn/Docum+/Runtwiss.man

To Print from rapt: (To Room 218 AGS 2nd Floor)

$$
\begin{gathered}
\text { alias it. 'cat * | psroff -t -ms > ppp; lp ppp' } \\
\text { it. } \quad \text { Manual Name }
\end{gathered}
$$



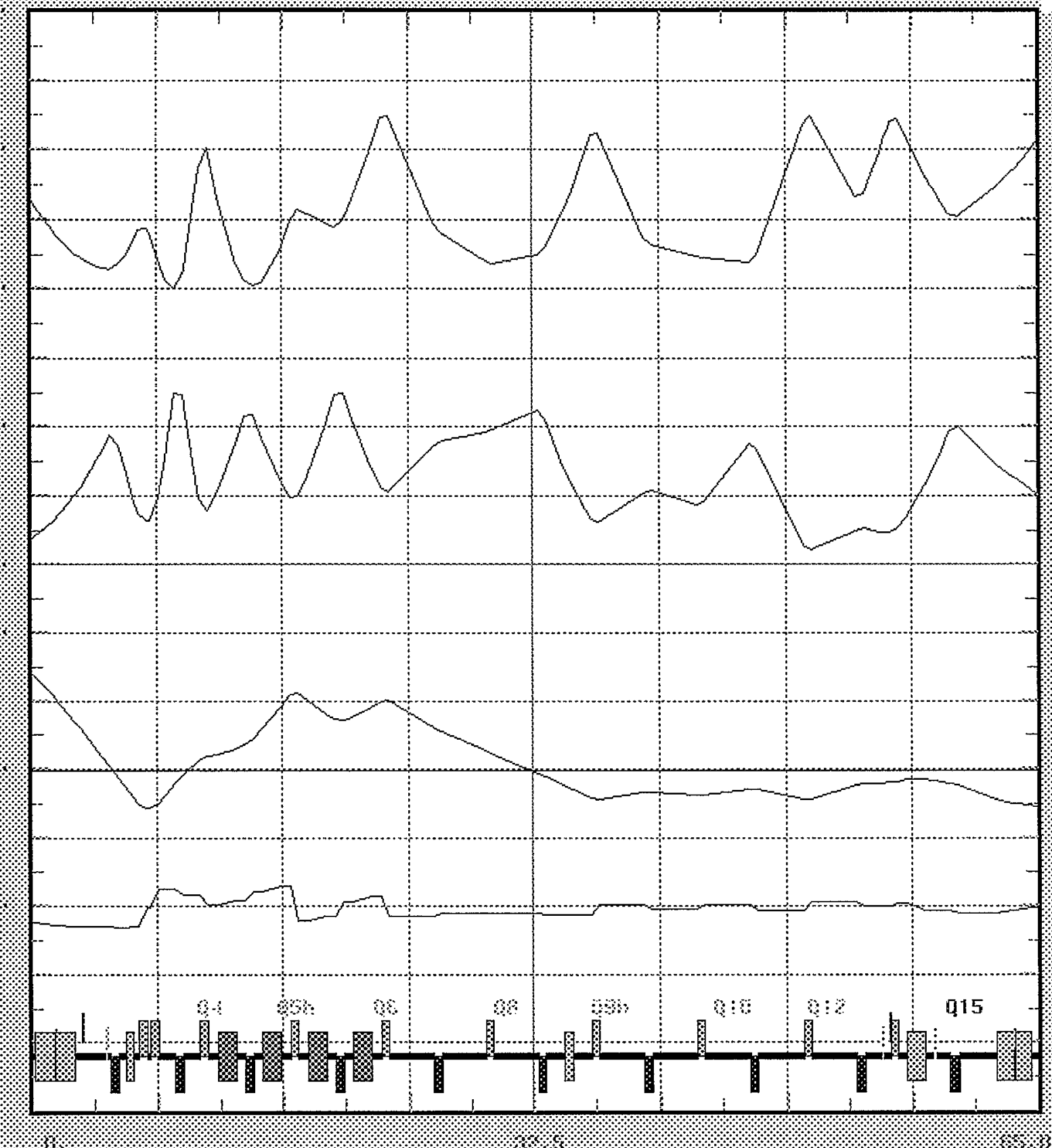


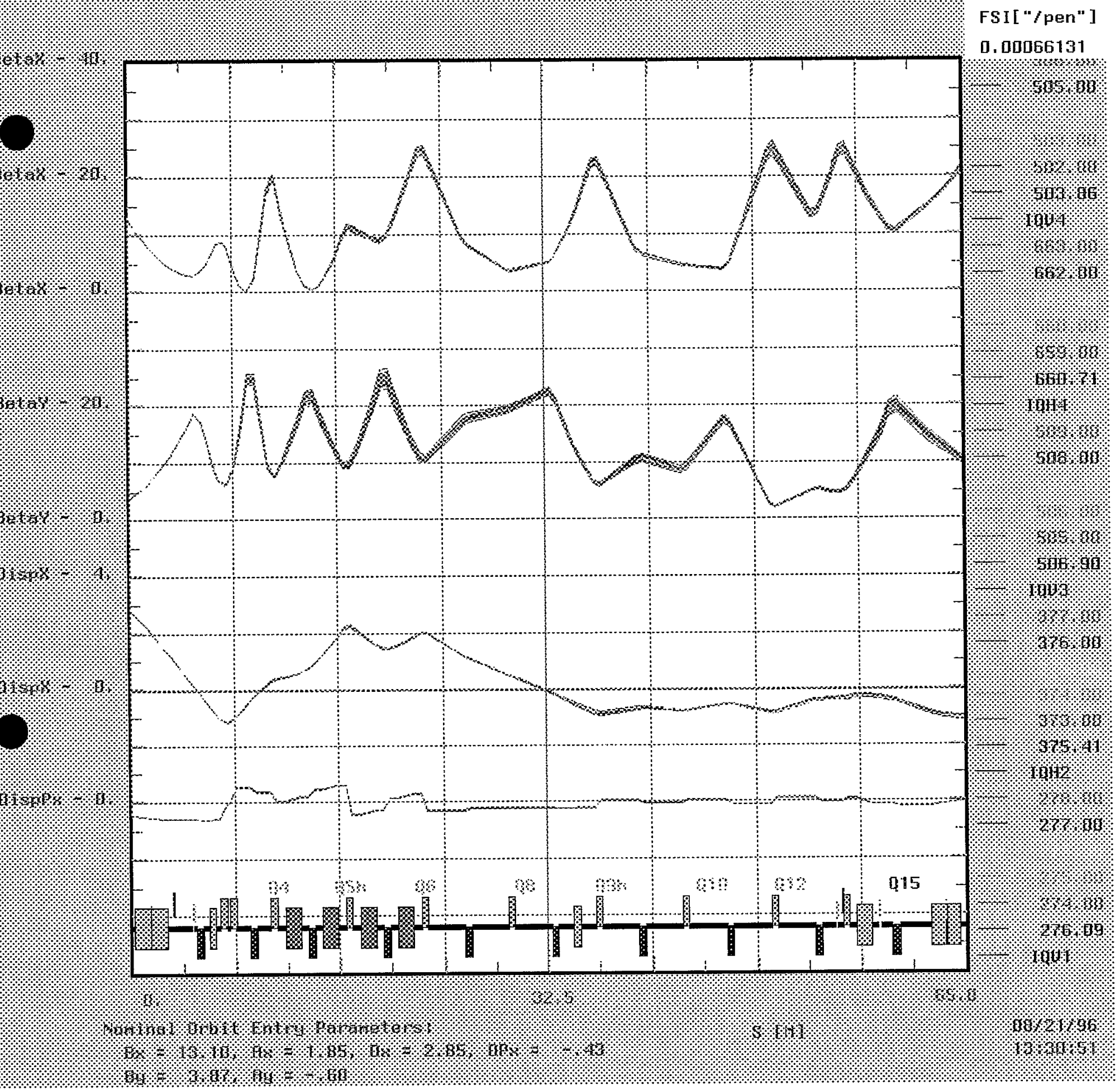

Figure 2. Sensitivity to -2 Amp through +2 Amp quad current drifts. 


$$
\text { ELEMENT SEQUENCE }
$$

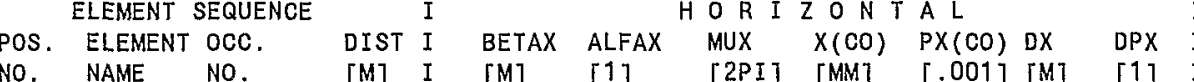
[2PI] [MM] [.001] [M] [1] I BEGIN JBTA

1 MQHF6

3 DHFGA

4 DRFGA1

5 DHFGT

6 DRF6A2

7 DHF6B

8 DRF6B

END LO

9 PUEHOO1

10 DROO1

11 MWOOE

12 DRDO6

13 DVO07

14 DROO7

15 QV1

16 DRQ1

$17 \mathrm{DH} 1$

END L1

EGIN JJ2

18 DRD1

19 QH2A

20 DRQ2A

21 QH2B

22 DRQ2B

23 XF019

24 DR019A

25 QV 3

26 DRQ3A

27 FOILO24

$28 \mathrm{DRO2}$

END JJ2

29 QH4

$30 \mathrm{JDQH} 4$

31 NBH2A

$32 \mathrm{JDH} 2 \mathrm{~A}$

33 NQV4

34 JDQV4

$35 \mathrm{NBH} 2 \mathrm{~B}$

$36 \mathrm{JDH} 2 \mathrm{~B}$

37 NQH5

38 JDQH5

39 NBH3A

40 JDH3A $\begin{array}{lll}0.000 & 13.101 & 1.851\end{array}$

$\begin{array}{lll}0.000 & 13.101 & 1.851 \\ 0.000 & 13.101 & 1.851\end{array}$

$\begin{array}{lll}0.236 & 12.246 & 1.771\end{array}$

$\begin{array}{lll}1.486 & 8.297 & 1.348\end{array}$

$\begin{array}{lll}1.506 & 8.244 & 1.341\end{array}$

1.506

1.526

2.853

3.259

3.259

3.259

3.259

4.344

4.344

4.776

5.005

5.166

5.724

6.128

6.661

6.661

6.661
6.661

7.040

7.599

7.715

8.274

8.734

8.734

8.734

9.324

9.883

9.989

9.989

10.933

10.933

10.933

11.491

12.091
13.301

13.301
13.901

14.400

15.000

16.210

16.810

17.308

17.908
19.118

19.118
19.718

$8.244 \quad 1.341$

$8.191 \quad 1.335$

$\begin{array}{ll}5.251 & 0.885\end{array}$

$\begin{array}{ll}4.588 & 0.747 \\ 4.588 & 0.747\end{array}$

$\begin{array}{lll}4.588 & 0.747\end{array}$

$\begin{array}{lll}4.588 & 0.747\end{array}$

$\begin{array}{ll}3.368 & 0.378\end{array}$

$\begin{array}{ll}3.368 & 0.378\end{array}$

$3.104 \quad 0.231$

$\begin{array}{ll}.975 & 0.099 \\ 3.634 & -1.361\end{array}$

$4.859-1.677$

$6.871-2.096$

$6.871-2.096$

$6.871-2.096$

$\begin{array}{lll}8.574 & -2.393\end{array}$

$8.732 \quad 2.138$

$\begin{array}{ll}8.246 & 2.064 \\ 4.374 & 4.182\end{array}$

$1.421 \quad 2.238$

$1.421 \quad 2.238$

$\begin{array}{lll}0.252 & -0.257\end{array}$

$2.232-3.736$

$3.101-4.447$

$3.101-4.447$

$17.467-10.772$

$17.467-10.772$

$20.336 \quad 6.517$

$13.286 \quad 5.234$

$3.737 \quad 2.665$

$\begin{array}{ll}1.320 & 1.364 \\ 0.592 & 0.167\end{array}$

$\begin{array}{ll}1.592 & 0.167 \\ 1.017 & -0.875\end{array}$

$5.643-2.953$

$9.806-3.986$

$\begin{array}{lll}11.600 & 0.657\end{array}$

$10.856 \quad 0.583$

9.5820 .471

$9.062 \quad 0.395$
$3.016 \quad 0.154$

$\begin{array}{llll}0.000 & 0.000 & 0.000 & 2.850-0.427\end{array}$

$\begin{array}{llll}0.000 & 0.000 & 0.000 & 2.850-0.427\end{array}$

$\begin{array}{llll}0.000 & 0.000 & 0.000 & 2.850-0.427\end{array}$

$\begin{array}{lllll}0.003 & 0.000 & 0.000 & 2.749-0.427\end{array}$

$\begin{array}{lllll}0.023 & 0.000 & 0.000 & 2.160-0.507\end{array}$

$\begin{array}{llll}0.023 & 0.000 & 0.000 & 2.150-0.507\end{array}$

$\begin{array}{llll}0.023 & 0.000 & 0.000 & 2.150-0.507\end{array}$

$\begin{array}{llll}0.023 & 0.000 & 0.000 & 2.140-0.507\end{array}$

$\begin{array}{llll}0.056 & 0.000 & 0.000 & 1.425-0.572\end{array}$

$\begin{array}{llll}0.069 & 0.000 & 0.000 & 1.193-0.572 \\ 0.069 & 0.000 & 0.000 & 1.193-0.572\end{array}$

$\begin{array}{llll}0.069 & 0.000 & 0.000 & 1.193-0.572 \\ 0.069 & 0.000 & 0.000 & 1.193-0.572\end{array}$

$\begin{array}{llll}0.069 & 0.000 & 0.000 & 1.193-0.572\end{array}$

$\begin{array}{llll}0.114 & 0.000 & 0.000 & 0.573-0.572\end{array}$

$\begin{array}{lllll}0.114 & 0.000 & 0.000 & 0.573-0.572\end{array}$

$\begin{array}{lllll}0.135 & 0.000 & 0.000 & 0.326-0.572\end{array}$

$\begin{array}{llll}0.147 & 0.000 & 0.000 & 0.195-0.572\end{array}$

$\begin{array}{llll}0.155 & 0.000 & 0.000 & 0.103-0.572 \\ 0.184 & 0.000 & 0.000 & -0.216-0.593\end{array}$

$\begin{array}{llll}0.199 & 0.000 & 0.000 & -0.455-0.593\end{array}$

$\begin{array}{lllll}0.214 & 0.000 & 0.000 & -0.760-0.553\end{array}$

$\begin{array}{lllll}0.214 & 0.000 & 0.000 & -0.760-0.553\end{array}$

$\begin{array}{lllll}0.214 & 0.000 & 0.000 & -0.760-0.553\end{array}$

$\begin{array}{lllll}0.221 & 0.000 & 0.000 & -0.970-0.553\end{array}$

$\begin{array}{llllll}0.231 & 0.000 & 0.000 & -1.127 & 0.004\end{array}$

$\begin{array}{llllll}0.233 & 0.000 & 0.000 & -1.127 & 0.004\end{array}$

$\begin{array}{llllll}0.233 & 0.000 & 0.000 & 0.000 & -0.965 & 0.559 \\ 0.277 & 0.000 & 0.000 & -0.708 & 0.559\end{array}$

$\begin{array}{llllll}0.277 & 0.000 & 0.000 & -0.708 & 0.559\end{array}$

$\begin{array}{llllll}0.500 & 0.000 & 0.000 & -0.378 & 0.559\end{array}$

$\begin{array}{llllll}0.646 & 0.000 & 0.000 & -0.120 & 0.391\end{array}$

$\begin{array}{llllll}0.653 & 0.000 & 0.000 & -0.079 & 0.391\end{array}$

$\begin{array}{llllll}0.653 & 0.000 & 0.000 & -0.079 & 0.391\end{array}$

$\begin{array}{llllll}0.673 & 0.000 & 0.000 & 0.290 & 0.391\end{array}$

$\begin{array}{llllll}0.673 & 0.000 & 0.000 & 0.290 & 0.391\end{array}$

$\begin{array}{llllll}0.673 & 0.000 & 0.000 & 0.290 & 0.391\end{array}$

$\begin{array}{llllll}0.677 & 0.000 & 0.000 & 0.422 & 0.061\end{array}$

$\begin{array}{llllll}0.683 & 0.000 & 0.000 & 0.459 & 0.061\end{array}$

$\begin{array}{lllll}0.711 & 0.000 & 0.000 & 0.626 & 0.217\end{array}$

$\begin{array}{lllll}0.754 & 0.000 & 0.000 & 0.757 & 0.217\end{array}$

$\begin{array}{llllll}0.852 & 0.000 & 0.000 & 0.924 & 0.462\end{array}$

$\begin{array}{llllll}0.993 & 0.000 & 0.000 & 1.201 & 0.462\end{array}$

$\begin{array}{llllll}1.077 & 0.000 & 0.000 & 1.852 & 0.615\end{array}$

$\begin{array}{lllll}1.090 & 0.000 & 0.000 & 2.221 & 0.615\end{array}$

$\begin{array}{llll}1.097 & 0.000 & 0.000 & 2.274-0.402\end{array}$

$\begin{array}{llll}1.105 & 0.000 & 0.000 & 2.033-0.402\end{array}$

$\begin{array}{llll}1.124 & 0.000 & 0.000 & 1.638-0.251\end{array}$

$\begin{array}{llll}1.135 & 0.000 & 0.000 & 1.488-0.251\end{array}$
BETAY ALFAY MERT I C A L

PY(CO) DY DPY | ELEMENT LENGTH STRENGTH $3.870-0.5960 .0000 .0000 .0000 .0000 .000$

$\begin{array}{lllllll}3.870 & -0.596 & 0.000 & 0.000 & 0.000 & 0.000 & 0.000\end{array}$

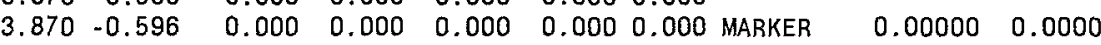
$\begin{array}{lllllllll}4.171 & -0.679 & 0.009 & 0.000 & 0.000 & 0.000 & 0.000 & \text { DRIFT }\end{array}$

$\begin{array}{lllllllll}0.415 & -1.090 & 0.048 & 0.000 & 0.000 & 0.000 & 0.000 & \text { SBEND }\end{array}$

$\begin{array}{llllllll}6.458 & -1.097 & 0.049 & 0.000 & 0.000 & 0.000 & 0.000 & \text { DRIFT }\end{array}$

$\begin{array}{llllllll}6.458 & -1.097 & 0.049 & 0.000 & 0.000 & 0.000 & 0.000 \\ \text { HKICK }\end{array}$

$\begin{array}{lllllll}6.501 & -1.104 & 0.049 & 0.000 & 0.000 & 0.000 & 0.000 \\ 0.9 R I F T\end{array}$

$\begin{array}{lllllll}9.999-1.531 & 0.075 & 0.000 & 0.000 & 0.000 & 0.000 & \text { SBEND }\end{array}$

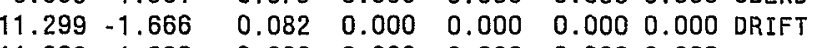

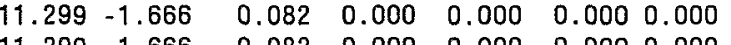

$11.299-1.666$

$11.299-1.666$

$15.308-2.029$

$15.308-2.029$

$17.126-2.174$

$18.138-2.250$

$18.869-2.304$

13.9814 .091

$.963 \quad 3.439$

$963 \quad 3.439$

$9.963 \quad 3.439$

$\begin{array}{ll}7.539 & 2.950\end{array}$

$6.411-0.741$

$6.586-0.769$

$9.802-5.531$

$15.571-7.014$

$15.571-7.014$

$24.973-8.916$

$24.889 \quad 9.047$

$23.007 \quad 8.693$

$23.007 \quad 8.693$

$9.559 \quad 5.551$

$9.559 \quad 5.551$

$9.559 \quad 5.55$

$10.663-2.511$

$17.540-3.131$

$21.519-3.501$

$21.807 \quad 2.951$

$18.426 \quad 2.684$

$9.792 \quad 1.989$

$9.972-2.376$

$13.063-2.776$

$20.514-3.337$

$24.732-3.692$

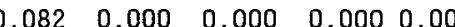

$\begin{array}{llllllll}0.082 & 0.000 & 0.000 & 0.000 & 0.000 & \text { MONITOR } & 0.00000 & 0.0000\end{array}$

$\begin{array}{llllllllll}0.095 & 0.000 & 0.000 & 0.000 & 0.000 & \text { DRIFT } & 1.08490 & 0.0000\end{array}$

\begin{tabular}{llllllll}
0.095 & 0.000 & 0.000 & 0.000 & 0.000 \\
\hline
\end{tabular}

$\begin{array}{llllllll}0.099 & 0.000 & 0.000 & 0.000 & 0.000 \text { DRIFT } & 0.43260 & 0.0000\end{array}$

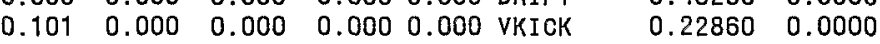

$\begin{array}{llllllll}0.102 & 0.000 & 0.000 & 0.000 & 0.000 & \text { DRIFT } & 0.16060 & 0.0000\end{array}$

$\begin{array}{lllllllll}0.107 & 0.000 & 0.000 & 0.000 & 0.000 & \text { QUADRUPO } & 0.55880 & -0.6755\end{array}$

$\begin{array}{llllllll}0.111 & 0.000 & 0.000 & 0.000 & 0.000 & \text { DRIFT } & 0.40315 & 0.0000\end{array}$

$\begin{array}{llllll}0.118 & 0.000 & 0.000 & 0.000 & 0.000 & \text { RBEND }\end{array}$

$0.53330 \quad 0.0364$

$\begin{array}{lllll}0.118 & 0.000 & 0.000 & 0.000 & 0.000\end{array}$

$\begin{array}{lllll}0.118 & 0.000 & 0.000 & 0.000 & 0.000\end{array}$

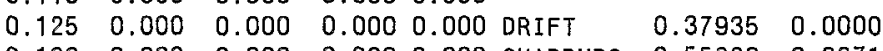

$\begin{array}{llllllll}0.139 & 0.000 & 0.000 & 0.000 & 0.000 & \text { QUADRUPO } & 0.55880 & 0.9271\end{array}$

$\begin{array}{lllllllll}0.142 & 0.000 & 0.000 & 0.000 & 0.000 & \text { DRIFT } & 0.11580 & 0.0000\end{array}$

$\begin{array}{llllllll}0.153 & 0.000 & 0.000 & 0.000 & 0.000 & \text { QUADRUPO } & 0.55880 & 0.9271\end{array}$

$\begin{array}{llllllll}0.159 & 0.000 & 0.000 & 0.000 & 0.000 & \text { DRIFT } & 0.45990 & 0.0000\end{array}$

$\begin{array}{llllllll}0.159 & 0.000 & 0.000 & 0.000 & 0.000 & \text { MARKER } & 0.00000 & 0.0000\end{array}$

$\begin{array}{lllllllll}0.164 & 0.000 & 0.000 & 0.000 & 0.000 & \text { DRIFT } & 0.59020 & 0.0000\end{array}$

$\begin{array}{lllllllll}0.167 & 0.000 & 0.000 & 0.000 & 0.000 & \text { QUADRUPO } & 0.55880 & -1.2487\end{array}$

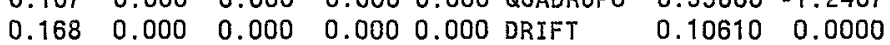

\begin{tabular}{llllllll}
0.168 & 0.000 & 0.000 & 0.000 & 0.000 \\
\hline
\end{tabular}

$\begin{array}{llllllll}0.178 & 0.000 & 0.000 & 0.000 & 0.000 & \text { DRIFT } & 0.94400 & 0.0000\end{array}$

$\begin{array}{lllll}0.178 & 0.000 & 0.000 & 0.000 & 0.000\end{array}$

$\begin{array}{llllllll}0.178 & 0.000 & 0.000 & 0.000 & 0.000 & & & \\ 0.189 & 0.000 & 0.000 & 0.000 & 0.000 & \text { QUADRUPO } & 0.55880 & 1.5902\end{array}$

$\begin{array}{llllllll}0.200 & 0.000 & 0.000 & 0.000 & 0.000 & \text { DRIFT } & 0.60000 & 0.0000\end{array}$

$\begin{array}{llllllll}0.214 & 0.000 & 0.000 & 0.000 & 0.000 & \text { SBEND } & 1.21000 & 0.1443\end{array}$

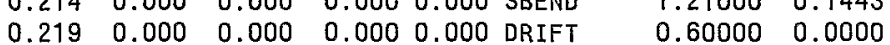

$\begin{array}{llllllll}0.222 & 0.000 & 0.000 & 0.000 & 0.000 & 0.04 A D R U P O & 0.49850 & -0.5923\end{array}$

$\begin{array}{llllllll}0.227 & 0.000 & 0.000 & 0.000 & 0.000 & \text { DRIFT } & 0.60000 & 0.0000\end{array}$

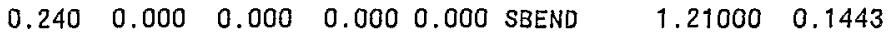

$\begin{array}{lllllllll}0.249 & 0.000 & 0.000 & 0.000 & 0.000 & \text { DRIFT } & 0.60000 & 0.0000\end{array}$

$\begin{array}{lllllll}0.257 & 0.000 & 0.000 & 0.000 & 0.000 \text { QUADRUPO } 0.49850 \quad 0.8912\end{array}$

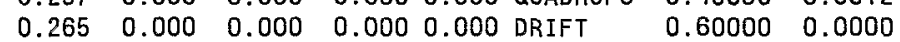

$\begin{array}{lllllll}0.277 & 0.000 & 0.000 & 0.000 & 0.000 \text { SBEND } & 1.21000 & 0.1443\end{array}$

$\begin{array}{lllllll}0.281 & 0.000 & 0.000 & 0.000 & 0.000 \text { DRIFT } & 0.60000 & 0.0000\end{array}$ 
ELEMENT SEQUENCE POS ELEMENT OCO. NO. NAME NO

\section{NQV5}

42 JDQV5

43 NBH3B

$44 \mathrm{JDH} 3 \mathrm{~B}$

45 NQH6

END JJ3

BEGIN JJ4

46
47 NQQH6

48 JDQV7

49 NQH8

50 JDQH8

51 NQY8

52 JDQH8A

$53 \mathrm{DH} 4$

$54 \mathrm{JDQHBB}$

END JJ4

BEGIN JJ5A

55 NQH9

56 JDQH9

57 NQV9

58 JDQV9

59 NQH1O

$60 \mathrm{JDQH} 10$

61 NQV11

62 JDQV11

63 NQH 12

64 JDQH12

END JJ5A

EEGIN JJ5B

65 QV13

66 DRQ13

67 MW166

68 DR166

69 DV168

70 DR168

71 PUEH170

72 DR170

$73 \mathrm{QH} 14$

74 DRQ14

75 DH5

END JJ5B

BEGIN L6

76 DRD5

77 DV181

78 DR181

79 XF183

80 DR183
I HOR I Z O N T A L

$x(C 0)$ PX(CO) DX [M] I [M] [1] [2PI] [MM] [.001] [M] [1] I

$\begin{array}{llllllll}20.217 & 9.984 & -2.328 & 1.143 & 0.000 & 0.000 & 1.464 & 0.156\end{array}$

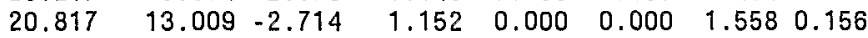
$\begin{array}{lllllllll}22.027 & 20.414 & -3.412 & 1.163 & 0.000 & 0.000 & 1.837 & 0.308\end{array}$ $\begin{array}{llllllll}22.627 & 24.731 & -3.784 & 1.168 & 0.000 & 0.000 & 2.022 & 0.308\end{array}$ $\begin{array}{lllllll}23.125 & 24.966 & 3.336 & 1.171 & 0.000 & 0.000 & 2.030-0.275\end{array}$ $\begin{array}{lllllll}23.125 & 24.966 & 3.336 & 1.171 & 0.000 & 0.000 & 2.030-0.275\end{array}$ $\begin{array}{lllllll}23.125 & 24.966 & 3.336 & 1.171 & 0.000 & 0.000 & 2.030-0.275\end{array}$ $\begin{array}{lrlllll}26.036 & 9.662 & 1.922 & 1.201 & 0.000 & 0.000 & 1.231-0.275\end{array}$ $\begin{array}{lllllll}26.534 & 8.173 & 1.099 & 1.210 & 0.000 & 0.000 & 1.115-0.190\end{array}$ $\begin{array}{llllllll}29.445 & 4.065 & 0.313 & 1.294 & 0.000 & 0.000 & 0.562-0.190\end{array}$ $\begin{array}{lllllll}29.943 & 3.812 & 0.194 & 1.314 & 0.000 & 0.000 & 0.467-0.192\end{array}$ $\begin{array}{llllllll}32.854 & 4.989 & -0.598 & 1.430 & 0.000 & 0.000 & -0.093-0.192\end{array}$

$\begin{array}{lllllll}33.352 & 6.147 & -1.794 & 1.445 & 0.000 & 0.000 & -0.194-0.218\end{array}$

$34.541-11.380-2.609 \quad 1.468$ 0.000 $0.000-0.453-0.218$

$\begin{array}{llllllll}35.074 & 14.358 & -2.975 & 1.474 & 0.000 & 0.000 & -0.564-0.199\end{array}$

$\begin{array}{lllllll}36.263 & 22.398 & -3.790 & 1.485 & 0.000 & 0.000 & -0.801-0.199\end{array}$

$\begin{array}{llllllll}36.263 & 22.398 & -3.790 & 1.485 & 0.000 & 0.000 & -0.801-0.199\end{array}$

$\begin{array}{llllllll}36.263 & 22.398 & -3.790 & 1.485 & 0.000 & 0.000 & -0.801-0.199\end{array}$

$\begin{array}{lllllllll}36.761 & 22.616 & 3.376 & 1.488 & 0.000 & 0.000 & -0.835 & 0.062\end{array}$

$\begin{array}{llll}39.672 & 7.609 & 1.781\end{array}$

40.170

43.081

43.579

46.490

46.988

49.899

50.397

53.307

53.307
53.307

53.806

54.403

54.403

54.833

55.077

55.374

55.374

55.492

55.990

56.497

57.743
57.743

57.743
57.743

58.194

58.194

58.438
58.818

58.818

59.344 $\begin{array}{lll}7.461 & 0.578\end{array}$

$\begin{array}{lll}4.844 & -0.023\end{array}$

$\begin{array}{lll}4.594 & 0.513\end{array}$

$3.938-0.287$

$\begin{array}{rr}4.971 & -1.890 \\ 23.765 & -4.567\end{array}$

$24.922 \quad 2.355$

$13.437 \quad 1.591$

$13.437 \quad 1.591$

$13.437 \quad 1.591$

$13.908-2.583$

$17.189-2.913$

$17.189-2.913$

$21.368-3.285$

$23.364-3.448$

$23.364-3.448$

$24.188-3.514$

$24.679 \quad 2.568$

$22.155 \quad 2.412$

$16.645 \quad 2.031$

$16.645 \quad 2.031$

$14.874 \quad 1.892$

$13.970 \quad 1.817$

$12.634 \quad 1.700$

$12.634 \quad 1.700$

$\begin{array}{llllll}1.524 & 0.000 & 0.000 & -0.654 & 0.062\end{array}$

$\begin{array}{lllll}1.535 & 0.000 & 0.000 & -0.647 & -0.034\end{array}$

$\begin{array}{llll}1.622 & 0.000 & 0.000 & -0.744-0.034\end{array}$

$\begin{array}{llllll}1.639 & 0.000 & 0.000 & -0.736 & 0.068\end{array}$

$\begin{array}{llllll}1.759 & 0.000 & 0.000 & -0.538 & 0.068\end{array}$

$\begin{array}{lllll}1.778 & 0.000 & 0.000 & -0.546-0.099\end{array}$

$\begin{array}{lllll}1.821 & 0.000 & 0.000 & -0.833-0.099\end{array}$

$\begin{array}{llllll}1.824 & 0.000 & 0.000 & -0.823 & 0.138 \\ 1.849 & 0.000 & 0.000 & -0.420 & 0.138\end{array}$

$1.8490 .000 \quad 0.000-0.4200 .138$

$\begin{array}{llllll}1.849 & 0.000 & 0.000 & -0.420 & 0.138\end{array}$

$\begin{array}{lllll}1.855 & 0.000 & 0.000 & -0.382 & 0.017\end{array}$

$\begin{array}{llllll}1.861 & 0.000 & 0.000 & -0.372 & 0.017\end{array}$

$\begin{array}{llllll}1.861 & 0.000 & 0.000 & -0.372 & 0.017\end{array}$

$\begin{array}{lllll}1.865 & 0.000 & 0.000 & -0.364 & 0.017 \\ 1.867 & 0.000 & 0.000 & -0.360 & 0.017\end{array}$

$\begin{array}{llllll}1.869 & 0.000 & 0.000 & -0.355 & 0.017\end{array}$

$\begin{array}{lllll}1.869 & 0.000 & 0.000 & -0.355 & 0.017\end{array}$

$\begin{array}{llllll}1.870 & 0.000 & 0.000 & -0.353 & 0.017\end{array}$

$\begin{array}{lllll}1.873 & 0.000 & 0.000 & -0.323 & 0.102\end{array}$

$\begin{array}{lllll}1.876 & 0.000 & 0.000 & -0.271 & 0.102\end{array}$

$\begin{array}{lllll}1.887 & 0.000 & 0.000 & -0.255-0.076\end{array}$

$\begin{array}{llll}1.887 & 0.000 & 0.000 & -0.255-0.076 \\ 1.887 & 0.000 & 0.000 & -0.255-0.076\end{array}$

$\begin{array}{llll}1.891 & 0.000 & 0.000 & -0.290-0.076\end{array}$

$\begin{array}{llll}1.894 & 0.000 & 0.000 & -0.308-0.076\end{array}$

$\begin{array}{lllll}1.899 & 0.000 & 0.000 & -0.337-0.076\end{array}$

$\begin{array}{llll}1.899 & 0.000 & 0.000 & -0.337-0.076\end{array}$

$\begin{array}{llll}1.906 & 0.000 & 0.000 & -0.377-0.076\end{array}$
VERTICAL

BETAY ALFAY MUY $Y(C O)$ PY(CO) DY DPY | ELEMENT LENGTH STRENGTH [M] [1] [2PI] [MM] [.001] [M] [1]

$\begin{array}{llllllllll}24.940 & 3.294 & 0.284 & 0.000 & 0.000 & 0.000 & 0.000 & \text { QUADRUPO } & 0.49850 & -0.5594\end{array}$ $\begin{array}{llllllllll}21.158 & 3.009 & 0.289 & 0.000 & 0.000 & 0.000 & 0.000 & \text { DRIFT } & 0.60000 & 0.0000\end{array}$ $\begin{array}{llllllllll}14.316 & 2.605 & 0.300 & 0.000 & 0.000 & 0.000 & 0.000 & \text { SBEND } & 1.21000 & 0.1443\end{array}$ $\begin{array}{llllllllll}11.386 & 2.279 & 0.307 & 0.000 & 0.000 & 0.000 & 0.000 & \text { DRIFT } & 0.60000 & 0.0000\end{array}$

$\begin{array}{llllllllll}10.724 & -0.890 & 0.314 & 0.000 & 0.000 & 0.000 & 0.000 & \text { QUADRUPO } & 0.49850 & 0.5696\end{array}$ $\begin{array}{llllllll}10.724 & -0.890 & 0.314 & 0.000 & 0.000 & 0.000 & 0.000\end{array}$

$\begin{array}{llllllll}10.724 & -0.890 & 0.314 & 0.000 & 0.000 & 0.000 & 0.000\end{array}$

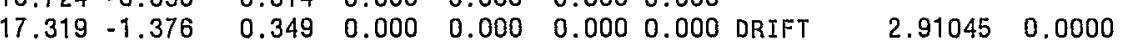
$\begin{array}{lllllllllll}18.083 & -0.137 & 0.353 & 0.000 & 0.000 & 0.000 & 0.000 & \text { QUADRUPO } & 0.49850 & -0.1450\end{array}$ $\begin{array}{llllllllll}19.359 & -0.301 & 0.378 & 0.000 & 0.000 & 0.000 & 0.000 & \text { DRIFT } & 2.91045 & 0.0000\end{array}$ $\begin{array}{llllllllll}19.714 & -0.411 & 0.382 & 0.000 & 0.000 & 0.000 & 0.000 & \text { QUADRUPO } & 0.49850 & 0.0083\end{array}$ $\begin{array}{llllllllll}22.610 & -0.584 & 0.404 & 0.000 & 0.000 & 0.000 & 0.000 & \text { DRIFT } & 2.91045 & 0.0000\end{array}$ $\begin{array}{lllllllllll}21.224 & 3.282 & 0.408 & 0.000 & 0.000 & 0.000 & 0.000 & \text { QUADRUPO } & 0.49850 & -0.3573\end{array}$ $\begin{array}{llllllllll}14.207 & 2.623 & 0.419 & 0.000 & 0.000 & 0.000 & 0.000 & \text { DRIFT } & 1.18858 & 0.0000\end{array}$ $\begin{array}{llllllllll}11.563 & 2.333 & 0.425 & 0.000 & 0.000 & 0.000 & 0.000 \text { RBEND } & 0.53330 & 0.0171\end{array}$

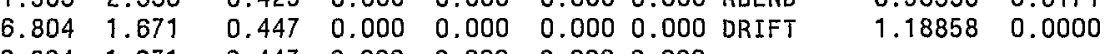
$6.804 \quad 1.671$ $6.804 \quad 1.671$

$6.231-0.460$

$.559-1.027$

$\begin{array}{ll}10.818 & 0.519\end{array}$

8.7890 .178

$9.244-1.112$

$17.764-1.816$

$16.833 \quad 3.587$

$.931 \quad 1.189$

$065-1.139$

$5.065-1.139$

$5.065-1.139$

.4620 .384

$5.078 \quad 0.258$

$5.078 \quad 0.258$

$4.895 \quad 0.168$

$\begin{array}{lll}4.825 & 0.117\end{array}$

$\begin{array}{ll}4.775 & 0.054\end{array}$

$\begin{array}{lll}4.775 & 0.054\end{array}$

$4.765 \quad 0.030$

$.399-1.354$

$6.906-1.620$

$11.524-2.038$

$11.524-2.038$

$11.524-2.038$

$13.456-2.240$

$14.575-2.349$

$16.424-2.519$

$16.424-2.519$

$19.195-2.754$

$\begin{array}{lllll}0.447 & 0.000 & 0.000 & 0.000 & 0.000 \\ 0.447 & 0.000 & 0.000 & 0.000 & 0.000\end{array}$

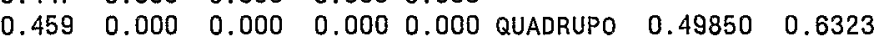

$\begin{array}{lllllllll}0.517 & 0.000 & 0.000 & 0.000 & 0.000 & \text { DRIFT } & 2.91045 & 0.0000\end{array}$

$\begin{array}{llllllll}0.525 & 0.000 & 0.000 & 0.000 & 0.000 & \text { QUADRUPO } & 0.49850 & -0.2976\end{array}$

$\begin{array}{llllllll}0.573 & 0.000 & 0.000 & 0.000 & 0.000 & \text { DRIFT } & 2.91045 & 0.0000\end{array}$

$\begin{array}{lllllllll}0.582 & 0.000 & 0.000 & 0.000 & 0.000 & 0.04 A D R U P O & 0.49850 & 0.2735\end{array}$

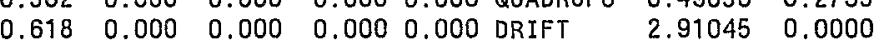

$\begin{array}{lllllllll}0.623 & 0.000 & 0.000 & 0.000 & 0.000 & \text { QUADRUPO } & 0.49850 & -0.6246\end{array}$

$\begin{array}{llllllll}0.691 & 0.000 & 0.000 & 0.000 & 0.000 & \text { DRIFT } & 2.91045 & 0.0000\end{array}$

$\begin{array}{llllllll}0.723 & 0.000 & 0.000 & 0.000 & 0.000 & \text { QUADRUPO } & 0.49850 & 0.5677\end{array}$

$\begin{array}{llllllll}0.886 & 0.000 & 0.000 & 0.000 & 0.000 & \text { DRIFT } & 2.91045 & 0.0000\end{array}$

$\begin{array}{llllll}0.886 & 0.000 & 0.000 & 0.000 & 0.000\end{array}$

$\begin{array}{llllll}0.886 & 0.000 & 0.000 & 0.000 & 0.000\end{array}$

$\begin{array}{llllllll}0.901 & 0.000 & 0.000 & 0.000 & 0.000 & \text { QUADRUPO } & 0.49850 & -0.6135\end{array}$

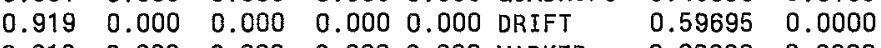

$\begin{array}{llllllll}0.919 & 0.000 & 0.000 & 0.000 & 0.000 & \text { MARKER } & 0.00000 & 0.0000\end{array}$

$\begin{array}{llllllllll}0.933 & 0.000 & 0.000 & 0.000 & 0.000 & \text { DRIFT } & 0.43050 & 0.0000\end{array}$

$\begin{array}{lllllllll}0.941 & 0.000 & 0.000 & 0.000 & 0.000 \\ \text { VKICK } & 0.24380 & 0.0000\end{array}$

$\begin{array}{lllllllll}0.951 & 0.000 & 0.000 & 0.000 & 0.000 & \text { DRIFT } & 0.29640 & 0.0000\end{array}$

$\begin{array}{lllllllll}0.951 & 0.000 & 0.000 & 0.000 & 0.000 & \text { MONITOR } & 0.00000 & 0.0000\end{array}$

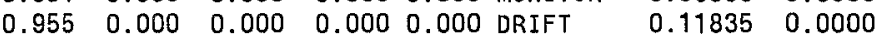

$\begin{array}{llllllll}0.971 & 0.000 & 0.000 & 0.000 & 0.000 & \text { QUADRUPO } & 0.49850 & 0.4962\end{array}$

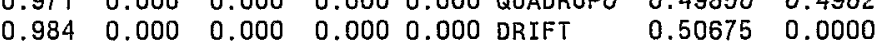

$\begin{array}{llllllll}1.006 & 0.000 & 0.000 & 0.000 & 0.000 & \text { RBEND } & 1.24560 & -0.1619\end{array}$

$\begin{array}{lllll}1.006 & 0.000 & 0.000 & 0.000 & 0.000\end{array}$

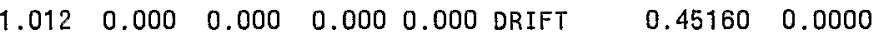

$\begin{array}{llllllll}1.015 & 0.000 & 0.000 & 0.000 & 0.000 & \text { VKICK } & 0.24380 & 0.0000\end{array}$

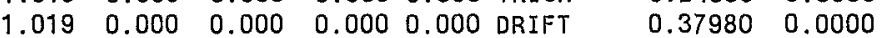

$\begin{array}{llllllll}1.019 & 0.000 & 0.000 & 0.000 & 0.000 \text { MARKER } & 0.00000 & 0.0000\end{array}$

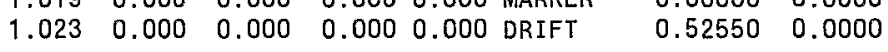


ELEMENT SEQUENCE

IST I BETAX ALFAX

R I Z O N T A L

$I$

VER T I C A L

\begin{tabular}{lllllllll|l} 
MUX & $X(C O)$ & $P X(C O)$ & $D X$ & $D P X$ & $I$ & BETAY ALFAY MUY $Y(C O)$ & PY(CO) DY & DPY & ELEMENT LENGTH STRENGTH
\end{tabular}

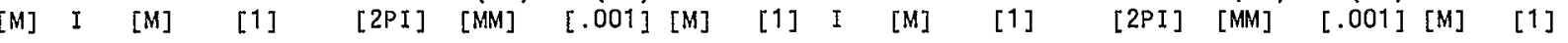

\begin{tabular}{|c|c|c|c|c|c|c|c|c|c|c|c|c|c|c|c|c|c|c|c|}
\hline 81 & QV15 & 1 & 59.902 & 10.471 & -0.681 & 1.914 & 0.000 & 0.000 & $-0.442-0.159$ & 20.079 & 1.233 & 1.028 & 0.000 & 0.000 & 0.000 & .000 & QUADRUPO & 0.55880 & -0.3641 \\
\hline 82 & DRQ15 & 1 & 60.139 & 10.800 & -0.714 & 1.918 & 0.000 & 0.000 & $-0.480-0.159$ & 19.503 & 1.203 & 1.030 & 0.000 & 0.000 & 0.000 & 0.000 & DRIFT & 0.23630 & 0.00 \\
\hline 83 & SHOLE & 1 & 60.139 & 10.800 & -0.714 & 1.918 & 0.000 & 0.000 & $-0.480-0.159$ & 19.503 & 1.203 & 1.030 & 0.000 & 0.000 & 0.000 & 0.000 & MARKER & .00000 & 000 \\
\hline END & L6 & 1 & 60.139 & 10.800 & -0.714 & 1.918 & 0.000 & 0.000 & $-0.480-0.159$ & 19.503 & 1.203 & 1.030 & 0.000 & 0.000 & 0.000 & 0.000 & & & \\
\hline BEGIN & L7 & 1 & 60.139 & 10.800 & -0.714 & 1.918 & 0.000 & 0.000 & $-0.480-0.159$ & 19.503 & 1.203 & 1.030 & 0.000 & 0.000 & 0.000 & 0.000 & & & \\
\hline 84 & DRQ15B & 1 & 62.401 & 14.745 & -1.030 & 1.946 & 0.000 & 0.000 & $-0.839-0.159$ & 14.702 & 0.919 & 1.051 & 0.000 & 0.000 & 0.000 & 0.000 & DRIFT & 2.26190 & 0.00 \\
\hline 85 & L20SPTM1 & 1 & 63.466 & 17.095 & -1.179 & 1.957 & 0.000 & 0.000 & $-0.969-0.087$ & 12.827 & 0.837 & 1.063 & 0.000 & 0.000 & 0.000 & 00 & RBEND & 514 & 0.065 \\
\hline 86 & DRL20A1 & 1 & 63.496 & 17.166 & -1.183 & 1.957 & 0.000 & 0.000 & $-0.972-0.087$ & 12.777 & 0.833 & 1.064 & 0.000 & 0.000 & 0.000 & 0.000 & DRIFT & 0.02987 & 0.000 \\
\hline 87 & L20SPTMT & 1 & 63.496 & 17.166 & -1.183 & 1.957 & 0.000 & 0.000 & $-0.972-0.087$ & 12.777 & 0.833 & 1.064 & 0.000 & 0.000 & 0.000 & 0.000 & HKICK & 0.00000 & 0.00 \\
\hline 88 & DRL20A2 & 1 & 63.525 & 237 & -1.187 & 1.958 & 0.000 & 0.000 & -0 . & 27 & 0.829 & 1.064 & 0.000 & 00 & 00 & 000 & DRIFT & 2987 & 0.000 \\
\hline 89 & L20SPTM2 & 1 & 64.560 & 19.840 & -1.331 & 1.967 & 0.000 & 0.000 & $8-0.017$ & 11.105 & 0.735 & 1.078 & 0.000 & 0.000 & 0.000 & 00 & RBEND & 454 & 0.063 \\
\hline 90 & DRL20B & 1 & 65.033 & 21.130 & -1.398 & 1.970 & 0.000 & 0.000 & $-1.036-0.017$ & 10.441 & 0.670 & 1.085 & 0.000 & 0.000 & 0.000 & 0.000 & DRIFT & 0.47272 & 0.000 \\
\hline END & L7 & 1 & 65.033 & 21.130 & -1.398 & 1.970 & 0.000 & 0.000 & $-1.036-0.017$ & 10.441 & 0.670 & 1.085 & 0.000 & 0.000 & 0.000 & 0.000 & & & \\
\hline END & JBTA & 1 & 65.033 & 21.130 & -1.398 & 1.970 & 0.000 & 0.000 & $-1.036-0.017$ & 10.441 & 0.670 & 1.085 & 0.000 & 0.000 & 0.000 & 0.000 & & & \\
\hline
\end{tabular}

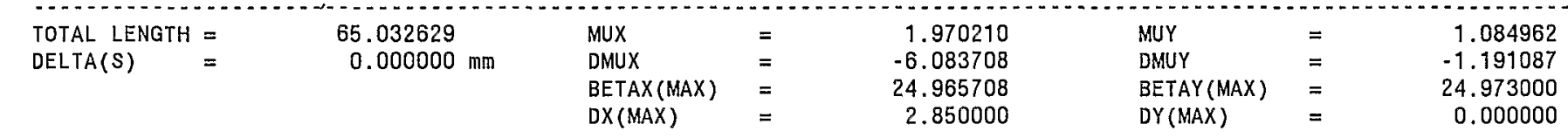

